\title{
Marcos legais da promoção da saúde no Brasil
}

\author{
Legal framework of health promotion in Brazil
}

Fernando Mussa Abujamra Aith

Aith FMA. Marcos legais da promoção da saúde no Brasil / Legal framework of health promotion in Brazil. Rev Med (São Paulo). 2013 abr.-jun.;92(2):148-54.

RESUMO: Apresentação e objetivos: A saúde foi reconhecida como um Direito humano social, expressamente previsto pela Constituição brasileira (arts. $\left.6^{\circ} \mathrm{e} 196\right)$ e por diversos instrumentos normativos internacionais. No campo de efetivação do direito à saúde, a promoção da saúde ocupa um lugar de destaque. $\mathrm{O}$ presente artigo tem como objetivo identificar os marcos legais internacionais e nacionais que atualmente norteiam a ação do Estado brasileiro no campo da promoção da saúde, bem como definem os direitos e deveres dos cidadãos no que se refere a este importante campo da saúde pública. Métodos: A metodologia adotada para a elaboração deste estudo foi a de pesquisa normativa aplicada, para fins de identificação dos marcos jurídicos e legais da promoção da saúde no Brasil. Resultados: Foram identificadas as normas internacionais que fazem referência expressa à promoção da saúde e, em seguida, as normas nacionais que apresentam dispositivos específicos sobre o tema. As normas nacionais foram relacionadas em ordem de importância hierárquica no campo jurídico, iniciando-se pela Constituição de 1988, seguindo-se as leis e, por fim, as normas infralegais (p.e., decretos, portarias, resoluções). Discussão e conclusões: A consolidação normativa da PNPS e de outras políticas associadas à promoção da saúde representa um avanço no desenvolvimento institucional do Estado brasileiro. No entanto, a edição formal de normas legais não é condição suficiente para que os seus ditames sejam cumpridos. Os desdobramentos práticos destas normas devem ser concretizados por ações e serviços públicos de promoção da saúde em todo o país. O acompanhamento vigilante da sociedade brasileira sobre o cumprimento das normas jurídicas editadas é essencial para o desenvolvimento do Brasil na área de promoção da saúde.

DESCRITORES: Promoção da saúde/legislação \& jurisprudência; Saúde/legislação \& jurisprudência; Direito à saúde/legislação \& jurisprudência; Brasil.

\begin{abstract}
Introduction and objectives: health was recognized as a social human right by the Brazilian Constitution (Articles 6 and 196) and by international normative instruments. To fulfill the right to health, health promotion occupies a prominent role. This article aims to identify the international and national legal frameworks that currently govern the action of the Brazilian State in the field of health promotion, as well as define the rights and duties of citizens in relation to health promotion. Methods: The methodology was to research rules about health promotion edited by international and national normative organisms. Results: We identified international legal documents that make explicit reference to health promotion as well as national laws and regulations that have specific provisions on the subject. National laws and regulations have been listed following the hierarchical importance in the legal field, starting with the 1988 Constitution, going through laws and finally identifying infralegal standards (eg, resolutions). Discussion and conclusions: The normative consolidation of National Policy on Health Promotion represents a breakthrough in the institutional development of the Brazilian state. However, the issue of formal legal rules is not sufficient condition for its fulfillment. The practical consequences of these legal frameworks must be achieved by providing actions and public health promotion services across the country. The accountability on the implementation of legal frameworks is essential for the development of Brazil in the area of health promotion.
\end{abstract}

KEYWORDS: Health promotion/legislation \& jurisprudence; Health/legislation \& jurisprudence; Right to health/legislation $\&$ jurisprudence; Brazil.

Professor Doutor do Departamento de Medicina Preventiva da Faculdade de Medicina da Universidade de São Paulo - FMUSP e do Núcleo de Pesquisa em Direito Sanitário da USP - NAP-DISA/USP.

Endereço para correspondência: Fernando Mussa Abujamra Aith. Av. Dr. Arnaldo, 455, $2^{\circ}$ andar, sala 2221. São Paulo, SP, Brasil. CEP: 01246-903. e-mail: fernando.aith@usp.br 


\section{INTRODUÇÃO}

A saúde foi reconhecida como um Direito humano social, expressamente previsto pela Constituição brasileira (artigos 6 e 196) ${ }^{1}$ e por diversos instrumentos normativos internacionais - notadamente a Constituição da Organização Mundial de Saúde ${ }^{2}$ e o Pacto dos Direitos Econômicos, Sociais e Culturais ${ }^{3}$. O reconhecimento da saúde como um Direito humano fundamental deu origem a uma profusão de normas jurídicas que têm o escopo de garantir esse importante direito.

Desde a Constituição Federal $^{1}$, passando por normas definidas em Tratados Internacionais, em leis internas e em normas infralegais brasileiras, encontraremos diversos instrumentos jurídico-normativos que tratam de variados aspectos relacionados com o direito à saúde, sempre voltados a garantir a saúde de cada indivíduo e da sociedade. $\mathrm{O}$ direito à saúde, reconhecido como um Direito Humano fundamental, pode ser categorizado no que se convencionou chamar de Direitos Sociais ou Direitos Humanos de segunda geração. A própria Constituição de 1988 expressamente declara a saúde como um direito social (artigo 6).

Cumpre destacar que o Direito à saúde, como direito social que é, realmente possui a característica de exigir do Estado brasileiro ações concretas e efetivas para a promoção, proteção e recuperação da saúde ${ }^{4}$. Deve assim o Estado intervir na dinâmica social para a proteção do Direito à saúde. De outro lado, a saúde também possui diversas características que lhe oferecem contornos de direito subjetivo público. O Direito à saúde pode ser também considerado como um Direito subjetivo público (faculdade de agir por parte de um cidadão ou de uma coletividade para ver um direito seu ser observado) na medida em que permite que o cidadão ingresse com uma ação junto ao Poder Judiciário para exigir do Estado, ou de terceiros responsáveis legalmente, a adoção ou a abstenção de medidas concretas em favor da saúde. A criação de leis e normas infralegais sobre o direito à saúde resulta no aprofundamento do dever estatal com relação a este direito, daí a importância em se compreender quais as normas legais que atualmente regulam a promoção da saúde no Brasil.

O Direito à saúde exige do Estado a adoção de ações concretas para sua promoção, proteção e recuperação, como a educação em saúde, a construção de hospitais, a adoção de programas de vacinação, a contratação de médicos, dentre ouras. Também se configura em um direito subjetivo público, ou seja, um direito oponível ao Estado por meio de ação judicial, pois permite que um cidadão ou uma coletividade exijam do Estado o fornecimento de um medicamento específico ou de um tratamento cirúrgico ou ainda de acesso a informações que sejam fundamentais para a promoção e proteção da saúde individual. O Direito à saúde é, portanto, um direito humano fundamental necessário para a proteção da dignidade humana e para o desenvolvimento do país.
Por essa razão as ações e serviços de saúde são, no Brasil, considerados como de relevância pública (artigo 197 da Constituição Federal) e devem estar sujeitos aos mecanismos de controle social de uma democracia participativa, capaz de evitar eventuais abusos a esse Direito ${ }^{1,5}$.

A plena realização do direito à saúde depende diretamente da atuação do Estado, já que a promoção, proteção e recuperação da saúde dependem, em larga medida, de uma determinada ação a ser tomada pelos órgãos estatais. E esta ação estatal deve ser, tanto quanto possível, legitimada por decisões tomadas com a participação da comunidade. Essas decisões são pautadas, sobretudo, por normas jurídicas internacionais, constitucionais, legais e infralegais que orientam as atividades do Estado.

A Constituição Federal ${ }^{1}$ prevê expressamente que as ações e serviços públicos de saúde integram uma rede regionalizada e hierarquizada e constituem um sistema único, organizado de acordo com as diretrizes de descentralização, atendimento integral e participação da comunidade (Art. 198, I, II, III). A participação da comunidade é, portanto, diretriz constitucional básica que deve ordenar as ações e serviços públicos de saúde. Ou seja, a gestão governamental das ações e serviços públicos de saúde deve dar-se dentro do que podemos chamar de Democracia Sanitária, uma forma de gestão da saúde pública onde o poder político é exercido pelo povo. O Estado democrático-constitucional tem historicamente articulado a convivência de um Direito com pretensão de legitimidade e um poder coercitivo que garante respaldo a esse Direito e, ao mesmo tempo, é por ele domesticado ${ }^{6}$.

A legitimidade democrática de uma norma encontrase estreitamente relacionada com a compreensão de quais as condições fundamentais para a preservação da dignidade e da autonomia privada e pública dos cidadãos ${ }^{6}$. A participação da sociedade na elaboração de normas jurídicas e, por vezes, da própria execução das políticas públicas que buscam dar concretude às normas jurídicas, elevada a princípio constitucional, mostra-se uma condição fundamental para a consolidação democrática no Brasil.

No campo de efetivação do direito à saúde, a promoção da saúde ocupa um lugar de destaque, desde o primeiro reconhecimento dado no âmbito do direito internacional até o reconhecimento oferecido pela Constituição brasileira e pelas demais normas jurídicas do Brasil. Seja por sua presença no próprio texto do artigo 196, seja pela presença nos documentos internacionais que o Brasil é signatário ou, ainda, seja pelas leis e normas infralegais editadas após 1988, a promoção da saúde, a sociedade e o Estado brasileiro deve atentar para a organização de uma rede de ações e serviços públicos de saúde capaz de cuidar deste aspecto fundamental da saúde humana, que é a promoção da saúde.

A promoção da saúde pode ser definida como o "processo de capacitação das pessoas e comunidades para modificarem os determinantes da saúde em benefício da 
própria qualidade de vida ${ }^{7}$. Ou, ainda, conforme consta da Carta de Ottawa ${ }^{8}$ :

"Promoção da saúde é o nome dado ao processo de capacitação da comunidade para atuar na melhoria de sua qualidade de vida e saúde, incluindo uma maior participação no controle deste processo. Para atingir um Estado de completo bem-estar físico, mental e social os indivíduos e grupos devem saber identificar aspirações, satisfazer necessidades e modificar favoravelmente o meio ambiente... Assim, a promoção da saúde não é responsabilidade exclusiva do setor saúde, e vai além de um estilo de vida saudável, na direção de um bem-estar global".

O presente artigo tem como objetivo identificar os marcos legais internacionais e nacionais que atualmente norteiam a ação do Estado brasileiro no campo da promoção da saúde, bem como definem os direitos e deveres dos cidadãos no que se refere a este importante campo da saúde pública.

\section{METODOLOGIA}

A metodologia adotada para a elaboração deste estudo foi a de pesquisa normativa aplicada, para fins de identificação dos marcos jurídicos e legais da promoção da saúde no Brasil. A pesquisa foi realizada com base na combinação das palavras-chave "promoção", "saúde", "direito à saúde" e "saúde pública, em bancos de dados oficiais da Organização das Nações Unidas (ONU), da Organização Mundial de Saúde (OMS), do Palácio do Planalto e do Ministério da Saúde. A busca foi realizada pelos sistemas de busca destes bancos de dados oficiais para fins de identificação das normas jurídicas direta ou indiretamente relacionadas com a promoção da saúde, editadas por estes entes e vigentes no Brasil.

As normas jurídicas encontradas foram analisadas em seu inteiro teor, para se identificar as que efetivamente possuem relação com a promoção da saúde no Brasil. As normas relacionadas neste estudo são as que apresentaram, após a análise, conteúdos que fazem referência direta à promoção da saúde.

\section{RESULTADOS}

Verificou-se que existem muitas normas jurídicas internacionais e nacionais que tratam da promoção da saúde, com maior ou menor detalhamento, de forma direta ou indireta. A promoção da saúde aparece com maior detalhamento nas normas jurídicas criadas para tratar de atenção primária à saúde, políticas públicas intersetoriais, saúde em todas as políticas ou ainda, no Brasil, de aspectos específicos relacionados com o funcionamento operacional do Sistema Único de Saúde e com o Sistema de Saúde Suplementar.

Para o presente artigo foram selecionadas as nor- mas jurídicas internacionais e nacionais que apresentam, de alguma forma, dispositivos que se referiam expressa e diretamente à promoção da saúde.

Foram identificadas as principais normas internacionais que fazem referência expressa à promoção da saúde e, em seguida, as normas nacionais que apresentam dispositivos específicos sobre o tema. As normas nacionais foram relacionadas em ordem de importância hierárquica no campo jurídico, iniciando-se pela Constituição de $1988^{1}$, seguindo-se as leis e, por fim, as normas infralegais que cuidam da promoção da saúde no Brasil atualmente (p.e., decretos, portarias, resoluções).

\section{Constituição da Organização Mundial de Saúde de $1946^{2}$}

O primeiro e mais relevante instrumento internacional de proteção do direito à saúde que faz menção específica à promoção da saúde é a Constituição da Organização Mundial de Saúde - OMS, aprovada em 1946². Trata-se de um Tratado Internacional assinado pelo Brasil que orienta a atuação de todos os Estados Partes para a melhoria das condições de saúde em seus territórios e no mundo.

O preâmbulo da Constituição da OMS apresenta um conceito jurídico de saúde bastante abrangente, dispondo que "a saúde é um estado de completo bem-estar físico, mental e social, e não consiste apenas na ausência de doença ou de enfermidade". Em seguida, o documento afirma que gozar do melhor estado de saúde que é possível atingir constitui um dos direitos fundamentais de todo o ser humano, sem distinção de raça, de religião, de credo político, de condição econômica ou social e que "os resultados conseguidos por cada Estado na promoção e proteção da saúde são de valor para todos". Outra importante menção do preâmbulo deste documento com relação à promoção da saúde estabelece que "o desigual desenvolvimento em diferentes países no que respeita à promoção de saúde e combate às doenças, especialmente contagiosas, constitui um perigo comum"2.

\section{Pacto Internacional dos Direitos Econômicos, Sociais e Culturais de 1966 $^{3}$}

O Pacto Internacional dos Direitos Econômicos, Sociais e Culturais é um Tratado Internacional, assinado pelo Brasil em 1966 e ratificado pelo Congresso Nacional somente em $1992^{3,9}$.

Os Estados Partes signatários do Pacto reconhecem o direito de toda pessoa desfrutar o mais elevado nível possível de saúde física e mental, dispondo que os Estados deverão adotar, com o fim de assegurar o pleno exercício desse direito, medidas que se façam necessárias para assegurar a diminuição da mortalidade infantil, bem como o desenvolvimento são das crianças; a melhoria de 
todos os aspectos de higiene do trabalho e do meio ambiente; a prevenção e tratamento das doenças epidêmicas, endêmicas, profissionais e outras, bem como a luta contra essas doenças; a criação de condições que assegurem a todos assistência médica e serviços médicos em caso de enfermidade.

Estes dispositivos do Pacto foram melhor detalhados por instrumentos normativos internacionais aprovados já no âmbito da Organização Mundial de Saúde.

\section{Declaração Alma Ata de $\mathbf{1 9 7 8}^{10}$}

A Declaração de Alma $\mathrm{Ata}^{10}$, aprovada em setembro de 1978 ao final da Conferência Internacional sobre Cuidados Primários de Saúde, realizada em Alma-Ata, no Cazaquistão, apresentou os primeiros consensos globais sobre a Promoção da Saúde como elemento essencial das políticas de saúde a serem desenvolvidas pelos governos dos Estados.

O documento declara que "as pessoas têm o direito e dever de participar do planejamento e execução das ações de promoção da saúde" (item IV), e que "os governos têm o dever de promover ações de promoção de saúde nos países mais necessitados para termos um mundo econômica e socialmente adequado para a produção da vida" (item V). Nesse sentido, a atenção primaria é a solução para o desenvolvimento do espírito de justiça social. A Declaração de Alma Ata afirma, ainda, que todos os governos devem lançar e aplicar políticas e programas voltados ao nível primário de atenção e promoção da saúde" (item VIII) ${ }^{10}$.

\section{Conferências Internacionais sobre Promoção da Saúde: de Ottawa à Helsinque}

A partir da Conferência Internacional sobre Cuidados Primários de Saúde de Alma-Ata ${ }^{10}$, a Organização Mundial de Saúde organizou várias outras Conferências para tratar de temas relevantes relacionados com a atenção primária, com especial destaque para as Conferências Internacionais sobre Promoção da Saúde.

A I Conferência Internacional sobre Promoção da Saúde foi realizada em 1986, em Ottawa, Canadá, e ao final aprovou a Carta de Ottawa ${ }^{8}$, uma outra declaração internacional de grande relevância para a promoção da saúde. A Carta de Ottawa estabelece expressamente a promoção da saúde como fator fundamental de melhoria da qualidade de vida e da saúde das pessoas, relacionando a promoção da saúde aos fatores determinantes e condicionantes da saúde e também à construção de políticas públicas que garantam ambientes favoráveis, através da mudança dos modos de vida, de trabalho e de lazer.

Depois da I Conferência Internacional sobre Promoção da Saúde em Ottawa foram realizadas outras, sempre tendo os preceitos da Declaração de Alma-Ata e da Carta de Ottawa como grandes referências. Assim, foram realizadas: a II Conferência, em Adelaide, Austrália (1988); a III Conferência, em Sundsvall, Suécia (1991); a IV Conferência, em Jacarta, Indonésia (1997); a V Conferência, na Cidade do México, México (2000); a VI Conferência, em Bangkok, na Tailândia, em 2005; a VII Conferência, em Nairóbi, Quênia (2009). AVIII Conferência está programada para este ano de 2013 e será realizada em Helsinque, Finlândia. Cada uma destas Conferências aprovou ao seu final uma Declaração Internacional focada na Promoção da Saúde, atualizando os objetivos e desafios traçados inicialmente em Ottawa.

A Conferência de Helsinque tem como objetivos facilitar a troca de experiências sobre políticas públicas intersetoriais, melhorar a capacidade para a implementação da saúde em todas as políticas, atualizar as contribuições da promoção da saúde no campo da atenção primária em saúde e rever os progressos, impactos e resultados da promoção da saúde desde Ottawa ${ }^{11}$.

\section{Constituição da República Federativa do Brasil de $1988^{1}$}

No Brasil, o reconhecimento normativo da promoção da saúde como um tema a ser tratado pelo Estado e pela sociedade iniciou-se com as grandes reformas promovidas pela Constituição da República Federativa do Brasil, promulgada em 05 de outubro de $1988^{1}$ após um período de regime de governo militar no país.

A Constituição refere-se expressamente à promoção da saúde em seu artigo 196, dispondo que "a saúde é direito de todos e dever do Estado, garantido mediante políticas sociais e econômicas que visem à redução do risco de doença e de outros agravos e ao acesso universal e igualitário às ações e serviços para sua promoção, proteção e recuperação"1.

\section{Lei 8.080 de $1990^{12}$}

A lei 8.080, de 19 de setembro de $1990^{12}$, organiza o Sistema Único de Saúde no Brasil. Esta lei dispõe sobre as condições para a promoção, proteção e recuperação da saúde, a organização e o funcionamento dos serviços correspondentes e dá outras providências.

De acordo com o seu Art. $2^{\circ}$, "saúde é um direito fundamental do ser humano, devendo o Estado prover as condições indispensáveis ao seu pleno exercício", e o "dever do Estado de garantir a saúde consiste na formulação e execução de políticas econômicas e sociais que visem à redução de riscos de doenças e de outros agravos e no estabelecimento de condições que assegurem acesso universal e igualitário às ações e aos serviços para a sua promoção, proteção e recuperação" 12 .

Outro importante dispositivo desta lei associado à promoção da saúde é o Art. $3^{\circ}$, que dispõe que "a saúde tem como fatores determinantes e condicionantes, entre outros, a alimentação, a moradia, o saneamento básico, o meio 
ambiente, o trabalho, a renda, a educação, o transporte, o lazer e o acesso aos bens e serviços essenciais; os níveis de saúde da população expressam a organização social e econômica do País"12.

A Lei 8.080/90 também se refere expressamente à promoção da saúde ao organizar as competências e atribuições da União, dos Estados, do Distrito Federal e dos Municípios para a garantia da saúde no Brasil. Nesse sentido, o Art. 15 dispõe que a União, os Estados, o Distrito Federal e os Municípios devem elaborar normas técnicas e estabelecimento de padrões de qualidade para promoção da saúde do trabalhador e também elaborar normas técnico-científicas de promoção, proteção e recuperação da saúde ${ }^{12}$

\section{Portaria $n^{0}$ 687/GM/MS de $2006^{13}$}

A Portaria n ${ }^{\circ}$ 687/GM/MS ${ }^{13}$, de 30 de março de 2006, aprova a Política Nacional de Promoção da Saúde (PNPS), estabelecendo as bases da atuação do Estado Brasileiro no campo da promoção da saúde. Trata-se de uma norma infralegal de grande importância administrativa para o Estado brasileiro, já que orienta a atuação de todos os agentes públicos da área da saúde para as ações e serviços associados à promoção da saúde no Brasil ${ }^{13}$.

A PNPS tem como bases normativas orientadoras as seguintes normas: Portaria $\mathrm{n}^{\circ} 648 / \mathrm{GM} / \mathrm{MS}$, de 28 de março de 2006, que aprova a Política Nacional de Atenção Básica (PNAB); Portaria n 971/GM/MS, de 3 de maio de 2006, que aprova a Política Nacional de Práticas Integrativas e Complementares (PNPIC); Portaria $n^{\circ} 710$ / GM/MS, de 10 de junho de 1999, que aprova a Política Nacional de Alimentação e Nutrição (PNAN); Portaria $n^{\circ}$ 325/GM/MS, de 21 de fevereiro de 2008, que estabelece prioridades, objetivos e metas do Pacto pela Vida para 2008, os indicadores de monitoramento e avaliação do Pacto pela Saúde e as orientações, prazos e diretrizes para sua pactuação. Vê-se, portanto, que a promoção da saúde está associada, também internamente, com a atenção primária à saúde (atenção básica) e às políticas associadas à intersetorialidade.

A PNSP tem como objetivo geral "promover a qualidade de vida e reduzir vulnerabilidade e riscos à saúde relacionados aos seus determinantes e condicionantes - modos de viver, condições de trabalho, habitação, ambiente, educação, lazer, cultura, acesso a bens e serviços essenciais"13.

\section{Portaria Interministerial $\mathrm{n}^{\circ} \mathbf{1 . 0 1 0}$ de $2006^{14}$}

A Portaria Interministerial $\mathrm{n}^{\circ} 1.010$, de 8 de maio de 2006, foi editada pelos Ministros da Saúde e da Educação, para tratar da dupla carga de doenças a que estão submetidos os países onde a desigualdade social continua a gerar desnutrição entre crianças e adultos, agravando assim o quadro de prevalência de doenças infecciosas ${ }^{14}$.

A Portaria institui as diretrizes para a Promoção da Alimentação Saudável nas Escolas de educação infantil, fundamental e nível médio das redes pública e privada, em âmbito nacional, favorecendo o desenvolvimento de ações que promovam e garantam a adoção de práticas alimentares mais saudáveis no ambiente escolar. Nesse sentido, a Portaria define a promoção da alimentação saudável nas escolas com base em alguns eixos prioritários, em especial as ações de educação alimentar e nutricional, considerando os hábitos alimentares como expressão de manifestações culturais regionais e nacionais e o estímulo à produção de hortas escolares para a realização de atividades com os alunos e a utilização dos alimentos produzidos na alimentação ofertada na escola ${ }^{14}$.

\section{Decreto $\mathrm{n}^{\mathbf{0}} \mathbf{6} .286$ de $2007^{15}$}

O Decreto $n^{\circ}$ 6.286, de 5 de dezembro de 2007, publicado pela Presidência da República, institui no âmbito dos Ministérios da Educação e da Saúde, o Programa Saúde na Escola - PSE, com finalidade de contribuir para a formação integral dos estudantes da rede pública de educação básica por meio de ações de prevenção, promoção e atenção à saúde ${ }^{15}$.

Dentre outros objetivos, o Decreto pretende promover a comunicação entre escolas e unidades de saúde, assegurando a troca de informações sobre as condições de saúde dos estudantes e fortalecer a participação comunitária nas políticas de educação básica e saúde, nos três níveis de governo. As ações em saúde previstas no âmbito do PSE considerarão a atenção, promoção, prevenção e assistência, e serão desenvolvidas articuladamente com a rede de educação pública básica e em conformidade com os princípios e diretrizes do SUS $^{15}$.

\section{Portaria $n^{0}$. 1.409/GM/MS de $2007^{16}$}

Para melhor implantação da PNPS, a Portaria $\mathrm{n}^{\mathrm{o}}$. 1.409/GM/MS ${ }^{16}$, de 13 de junho de 2007, instituiu o Comitê Gestor da Política Nacional de Promoção da Saúde - CGPNPS com as atribuições de consolidar a implementação da Política Nacional de Promoção da Saúde e a Agenda Nacional de Promoção da Saúde. O Comitê Gestor tem ainda as funções de coordenar a implantação da Política Nacional de Promoção da Saúde no SUS e em sua articulação com os demais setores governamentais e não-governamentais e monitorar e avaliar as estratégias de implantação/implementação da Política Nacional de Promoção da Saúde e seu impacto na melhoria da qualidade de vida de sujeitos e coletividades ${ }^{16}$.

\section{Portaria Interministerial $\mathrm{n}^{0} .675$ de $2008^{17}$}

A Portaria Interministerial $\mathrm{N}^{\mathrm{o}} 675$, de 4 de junho 
de 2008, também foi editada pelos Ministérios da Saúde e Educação para instituir a Comissão Intersetorial de Educação e Saúde na Escola - CIESE, com a finalidade de estabelecer diretrizes da política de educação e saúde na escola, em conformidade com as políticas nacionais de educação e com os objetivos, princípios e diretrizes do Sistema Único de Saúde - SUS ${ }^{17}$.

\section{Portaria GM/MS no. 719 de $2011^{18}$}

A Portaria $n^{\circ} .719$, de 07 de abril de $2011^{18}$, institui o Programa Academia da Saúde no âmbito do Sistema Único de Saúde, a ser implantado pelas Secretarias de Saúde do Distrito Federal e dos Municípios, com o apoio técnico das Secretarias Estaduais de Saúde e do Ministério da Saúde. O Programa Academia da Saúde tem como objetivo principal contribuir para a promoção da saúde da população a partir da implantação de polos com infraestrutura, equipamentos e quadro de pessoal qualificado para a orientação de práticas corporais e atividade física e de lazer e modos de vida saudáveis. Conforme previsto na Portaria, os polos do Programa Academia da Saúde tem como objetivos específicos ampliar o acesso da população às políticas públicas de promoção da saúde; fortalecer a promoção da saúde como estratégia de produção de saúde; potencializar as ações nos âmbitos da Atenção Primária em Saúde (APS), da Vigilância em Saúde (VS) e da Promoção da Saúde (PS); promover a integração multiprofissional na construção e execução das ações; promover a convergência de projetos ou programas nos âmbitos da saúde, educação, cultura, assistência social, esporte e lazer; ampliar a autonomia dos indivíduos sobre as escolhas de modos de vida mais saudáveis; aumentar o nível de atividade física da população; estimular hábitos alimentares saudáveis; promover mobilização comunitária com a constituição de redes sociais de apoio e ambientes de convivência e solidariedade; potencializar as manifestações culturais locais e o conhecimento popular na construção de alternativas individuais e coletivas que favoreçam a promoção da saúde; e contribuir para ampliação e valorização da utilização dos espaços públicos de lazer, como proposta de inclusão social, enfrentamento das violências e melhoria das condições de saúde e qualidade de vida da população ${ }^{18}$.

\section{DISCUSSÃO}

Pelo levantamento normativo realizado percebe-se que é grande a importância dada à promoção da saúde no campo das políticas públicas, seja no âmbito internacional como no âmbito nacional.

No âmbito internacional, a promoção da saúde apresenta-se como elemento essencial das políticas públicas a serem realizadas pelos Estados para fins de garantia do direito à saúde ${ }^{2,3}$. Nas normas jurídicas levantadas, a promoção da saúde insere-se sobretudo no contexto da atenção primária em saúde ${ }^{10}$ e da intersetorialidade de políticas públicas voltadas ao bem estar físico, mental e social das pessoas ${ }^{2,8}$.

No âmbito nacional, a promoção da saúde foi alçada a um dever estatal, inserido no contexto da saúde como direito de todos e dever do Estado a ser garantido mediante políticas sociais e econômicas que visem à promoção, proteção e recuperação da saúde, conforme previsto no Art. 196 da Constituição Federal ${ }^{1 .}$

A Lei 8.080/90 representou um importante reforço para a consolidação da promoção da saúde como elemento essencial das políticas públicas de saúde no Brasil, ao prever que a União, os Estados, o Distrito Federal e os Municípios devem desenvolver ações e serviços de promoção da saúde ${ }^{12}$.

No que se refere à legislação infralegal, destaquese a publicação da Portaria n ${ }^{0}$ 687/GM/MS de $2006^{13}$, que instituiu formalmente no âmbito nacional, com impactos importantes para os demais entes federativos, a Política Nacional de Promoção da Saúde, definindo objetivos, princípios, diretrizes e estratégias de ação. Em 2007, a instituição do Comitê Gestor da PNPS também representou importante passo administrativo para a consolidação da promoção da saúde no Sistema de Saúde brasileiro. Nesse sentido, faz-se notar a ausência de representantes da sociedade civil entre os integrantes do Comitê Gestor da PNPS, o que contraria a diretriz de participação da comunidade no SUS, conforme estabelecido no artigo Art. 198, III, da Constituição Federal ${ }^{16}$.

Outro importante resultado encontrado foi a existência de Portarias Interministeriais e de um Decreto Presidencial estabelecendo importantes políticas intersetoriais no campo da promoção da saúde, o que representa um modelo de vanguarda na configuração das políticas públicas de saúde no Brasi1 ${ }^{17}$. Esta forma de construir políticas públicas para enfrentar as grandes questões de saúde da sociedade está em consonância com as mais modernas compreensões sobre como enfrentar os fatores determinantes da saúde, focadas na formulação e execução de políticas públicas intersetoriais e na ideia de saúde em todas as políticas, que é justamente a pauta da $8^{\mathrm{a}}$ Conferência Internacional em Promoção da Saúde ${ }^{11}$ que se realizará na Finlândia neste ano de 2013.

\section{CONSIDERAÇÕES FINAIS}

A pesquisa realizada foi relevante para identificar os marcos legais da promoção da saúde vigentes no Brasil atualmente. No contexto de um Estado Democrático de Direito, as leis e normas infralegais representam um importante ponto de partida sobre os consensos sociais estabelecidos formalmente acerca das mais variadas questões que afetam a sociedade. Conhecer a forma como o Estado brasileiro organiza juridicamente a promoção 
da saúde auxilia a compreender os atuais avanços já verificados na área bem como as fragilidades ainda a serem superadas.

A consolidação normativa da PNPS e de outras políticas associadas à promoção da saúde foi um importante avanço verificado no desenvolvimento institucional do Estado brasileiro. No entanto, a simples edição de uma norma legal não é condição suficiente para

\section{REFERÊNCIAS}

1. Brasil. Constituição da República Federativa do Brasil. Brasília; 1988 [citado 16 maio 2013]. Disponível em: http:// www.planalto.gov.br/ccivil_03/constituicao/ConstituicaoCompilado.htm.

2. World Health Organization (WHO). Constitution of the World Health Organization. Geneve; 1946 [cited 2013 May 16]. Available from: http://apps.who.int/gb/bd/PDF/bd47/EN/ constitution-en.pdf.

3. United Nations. Human Rights. Office of the Hight Commissioner for Human Rights. International Covenant on Economic, Social and Cultural Rights. Geneve; 1966 [cited 2013 May 16]. Available from: http://www.ohchr.org/EN/ ProfessionalInterest/Pages/CESCR.aspx.

4. Trindade AAC. Tratado de direito internacional dos direitos humanos. Porto Alegre: editora safE; 1997. v.1.

5. Bobbio N. Estado, governo, sociedade: para uma teoria geral da política. Trad. Marco Aurélio Nogueira. 9a ed. São Paulo: Paz e Terra; 2001.

6. Vieira OV. A constituição e sua reserva de justiça. São Paulo: Ed. Malheiros; 1999.

7. Candeias NMF. Conceitos de educação e de promoção em saúde: mudanças individuais e mudanças organizacionais. Rev Saude Publica. 1997;31(2):209-13. http://dx.doi. org/10.1590/S0034-89101997000200016

8. World Health Organization (WHO). The Ottawa Charter for Health Promotion. First International Conference on Health Promotion, Ottawa, 21 Nov. 1986. Geneve; 1986 [cited 2013 May 16]. Available from: http://www.who.int/ healthpromotion/conferences/previous/ottawa/en/.

9. Brasil.. Presidência da República. Decreto no 591, de 6 de julho de 1992. Brasília; 1992 [citado 16 maio 2013]. Disponível em: http://www.jusbrasil.com.br/legislacao/113459/ decreto-591-92.

10. World Health Organization (WHO). Declaration of alma-Ata International Conference on Primary Health CAre, AlmaAta, USRR, 6-12 Sept. 1978. Geneve; 1978 [cited 2013 que os seus ditames sejam cumpridos. Os desdobramentos práticos destas normas devem ser concretizados por ações e serviços públicos de promoção da saúde em todo o país, razão pela qual o acompanhamento vigilante da sociedade brasileira sobre o cumprimento das normas jurídicas editadas representa um fator importante para o desenvolvimento do Brasil na área de promoção da saúde.

May 16]. Available from: http://www.who.int/publications/ almaata_declaration_en.pdf.

11. World Health Organization (WHO). 8th Global Conference on Health Promotion - the Helsinki Statement on Health in All Policies. Geneva; 2013 [cited 2013 May 16]. Available from: http://www.who.int/healthpromotion/conferences/8gchp/en/ index.html

12. Brasil. Presidência da República. Lei $\mathrm{n}^{\circ} 8.080$, de 19 de setembro de 1990. Brasília; 1990 [citado 16 maio 2013]. Disponível em: http://portal.saude.gov.br/portal/arquivos/ pdf/ lei8080.pdf.

13. Brasil. Ministério da Saúde. Portaria $n^{\circ} 687 / \mathrm{GM} / \mathrm{MS}$, de 30 de março de 2006. Brasília; 2006 [citado 16 maio 2013]. Disponível em: http://portal.saude.gov.br/portal/arquivos/ pdf/pactovolume7.pdff.

14. Brasil. Ministério da Saúde. Portaria Interministerial n ${ }^{\circ} 1.010$, de 8 de maio de 2006. Brasília; 2006 [citado 16 maio 2013]. Disponível em: http://dtr2001.saude.gov.br/sas/PORTARIAS/ Port2006/GM/GM-1010.htm.

15. Brasil. Presidência da República. Decreto $n^{\circ} 6.286$, de 5 de dezembro de 2007.Brasília; 2007 [citado em 16 maio 2013]. Disponível em: http://www.planalto.gov.br/ccivil_03/ ato2007-2010/2007/decreto/d6286.htm.

16. Brasil. Ministério da Saúde. Portaria ${ }^{\circ}$. 1.409/GM/MS, de 13 de junho de 2007. Brasília; 2007 [citado 16 maio 2013]. Disponível em: http://bvsms.saude.gov.br/bvs/saudelegis/ gm/2007/prt1409_13_06_2007.html

17. Brasil. Ministério da Saúde. Portaria Interministerial No 675 , de 4 de Junho de 2008. Brasília; 2008 [citado 16 maio 2013]. Disponível em: bvsms.saude.gov.br/bvs/saudelegis/mec/ pri0675_04_06_2008.pdf.

18. Brasil. Ministério da Saúde. Portaria nº. 719, de 07 de abril de 2011. Brasília; 2011 [citado 16 maio 2013]. Disponível em: portal.saude.gov.br/portal/arquivos/.../portaria_academia_ saude_719.pdf. 